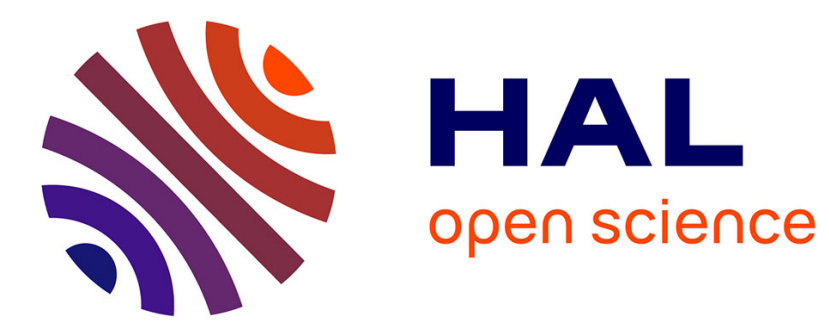

\title{
Resolved fine and hyperfine state-to-state rate coefficients for the rotational transitions of C3N induced by collision with $\mathrm{He}$
}

\author{
Miguel Lara-Moreno, Thierry Stoecklin, Philippe Halvick
}

\section{- To cite this version:}

Miguel Lara-Moreno, Thierry Stoecklin, Philippe Halvick. Resolved fine and hyperfine state-to-state rate coefficients for the rotational transitions of C3N induced by collision with He. Monthly Notices of the Royal Astronomical Society, 2021, 507 (3), pp.4086-4094. 10.1093/mnras/stab2453 . hal03406083

\section{HAL Id: hal-03406083 \\ https://hal.science/hal-03406083}

Submitted on 27 Oct 2021

HAL is a multi-disciplinary open access archive for the deposit and dissemination of scientific research documents, whether they are published or not. The documents may come from teaching and research institutions in France or abroad, or from public or private research centers.
L'archive ouverte pluridisciplinaire HAL, est destinée au dépôt et à la diffusion de documents scientifiques de niveau recherche, publiés ou non, émanant des établissements d'enseignement et de recherche français ou étrangers, des laboratoires publics ou privés. 


\title{
Resolved fine and hyperfine state-to-state rate coefficients for the rotational transitions of $\mathrm{C}_{3} \mathrm{~N}$ induced by collision with $\mathrm{He}$
}

\author{
Miguel Lara-Moreno, ${ }^{1}$ Thierry Stoecklin, ${ }^{1 \star}$ Philippe Halvick ${ }^{1}$ \\ ${ }^{1}$ Université de Bordeaux, ISM, UMR 5255, 33405, Talence, France
}

16 August 2021

\begin{abstract}
The fine and hyperfine resolved state-to-state rate coefficients for the rotational (de)excitation of $\mathrm{C}_{3} \mathrm{~N}$ by collision with helium are computed. To this aim a two dimensional potential energy surface is calculated for this system. The recoupling method is used to obtain the fine and hyperfine structure resolved rate coefficients from spin-free Close Coupling calculations. These results are compared with those given by the Infinite Order Sudden Approximation and the $M$-randomizing Limit. General propensity rules for the transitions are also found and analyzed.
\end{abstract}

Key words: scattering - molecular data - molecular processes - ISM: molecules

\section{Introduction}

Radicals play an important role in the chemical evolution of interstellar clouds. Among them, the cyanoethynyl radical $\mathrm{C}_{3} \mathrm{~N}$ was first detected in 1980 at the centers of the two dark clouds TMC 1 and TMC 2(Friberg et al. 1980). It recently received significant attention due to its possible role as a precursor in the formation of the detected $\mathrm{C}_{3} \mathrm{~N}^{-}$anion (Thaddeus et al. 2008) by radiative electron attachment (REA) (Lara-Moreno et al. 2019a). One of the most important parameter needed for assessing the REA mechanism is the anion-to-neutral abundance ratio. The magnitude of this ratio is obtained from the analysis of emission spectra which requires the knowledge of collisional excitation and de-excitation rate coefficients for both the charged and neutral species. Various studies have been dedicated to the determination of the collisional rates for the $\mathrm{C}_{3} \mathrm{~N}^{-}$anion (Lara-Moreno et al. 2017; Tchakoua et al. 2018; LaraMoreno et al. 2019b). However, despite of being a well know ISM species for a long time, there is no collisional data available for $\mathrm{C}_{3} \mathrm{~N}$. The present study is then dedicated to the calculation of state-tostate rate coefficients for the rotational transitions of $\mathrm{C}_{3} \mathrm{~N}$ induced by collision with $\mathrm{He}$, which could also serve as an approximation for rotational transitions induced by collision with para- $\mathrm{H}_{2}$.

The $A^{2} \Pi$ first excited electronic state of $\mathrm{C}_{3} \mathrm{~N}$ exhibits a low frequency bending mode and is subjected to the Renner-Teller effect. We can assume that it is only weakly coupled with the ${ }^{2} \Sigma$ ground state of $\mathrm{C}_{3} \mathrm{~N}$ owing to its excitation energy of $1844 \mathrm{~cm}^{-1}$ (Hoshina \& Endo 2007). Therefore, since we use the rigid-rotor approximation and we are interested in the rate coefficients at temperatures below $500 \mathrm{~K}$, we perform the calculations only with the electronic ground state of $\mathrm{C}_{3} \mathrm{~N}$.

$\mathrm{C}_{3} \mathrm{~N}\left(X^{2} \Sigma\right)$ is a tetra-atomic linear radical with a non-zero nu-

^ E-mail: thierry.stoecklin@u-bordeaux.fr clear spin. The fine and hyperfine structure need then to be considered. In the present study the hyperfine and fine resolved rate coefficients are obtained using the recoupling method. The recoupling results will also be compared with those obtained from the Infinite Order Sudden Approximation (IOSA) and the $M$-randomizing limit (MRL).

The manuscript is organized as follows. In section 2 the main steps of the methods are reviewed. The construction of the 2D PES is presented in section 2.1 while section 2.2 is dedicated to the presentation of scattering formalism, the recoupling method, the IOSA and MRL approximations. The results are reported and discussed in section 3 and the conclusion of this study is presented in section 4 .

\section{Methods}

\subsection{Potential Energy Surface}

\subsubsection{Ab-initio calculations}

Numerous studies have been dedicated to characterize the ground $X^{2} \Sigma^{+}$and low lying $A^{2} \Pi$ states of the $\mathrm{C}_{3} \mathrm{~N}$ radical. For a detailed discussion of these previous works, we refer the reader to McCarthy et al. (1995); Botschwina et al. (1993) and the references therein. In the present work, we use the restricted open shell coupled cluster method with single and double excitations using a perturbative treatment of triple excitations $(\operatorname{RCCSD}(\mathrm{T}))$ which offers a good compromise between cpu time and accuracy for describing the $\mathrm{C}_{3} \mathrm{~N}$ $\left(X^{2} \Sigma^{+}\right)$ground state. For the one-electron basis set, we selected the augmented correlation consistent polarized valence triple zeta (aug-cc-pVTZ) basis set complemented by a set of mid-bond functions (Cybulski \& Toczylowski 1999) which is known to provide a better description of the long-range van der Waals interaction. 
Interaction energies were first computed for a $2 \mathrm{D}$ grid of geometries of the He- $\mathrm{C}_{3} \mathrm{~N}$ complex expressed in Jacobi coordinates $(R, \theta)$, where $R$ stands for the intermolecular distance between $\mathrm{He}$ and the center of mass of $\mathrm{C}_{3} \mathrm{~N}$ while $\theta$ is the angle between the $\mathrm{C}_{3} \mathrm{~N}$ axis and the intermolecular axis, with $\theta=0$ corresponding to $\mathrm{He}$ facing the $\mathrm{N}$-end of $\mathrm{C}_{3} \mathrm{~N}$. All the $\mathrm{C}_{3} \mathrm{~N}$ bond distances were set to their equilibrium values in the ground state obtained from $\operatorname{CCSD}(\mathrm{T})$ calculations (Woon \& Herbst 2009; McCarthy et al. 1995), namely $r_{\mathrm{C}_{1}-\mathrm{C}_{2}}=2.2998 a_{0}, r_{\mathrm{C}_{2}-\mathrm{C}_{3}}=2.6134 a_{0}, r_{\mathrm{C}_{3}-\mathrm{N}}=2.2053 a_{0}$. Due to the expected strong anisotropy of the interaction, we chose a fine grid made of 37 angular steps with $\theta$ ranging from $0^{\circ}$ to $180^{\circ}$ and for each angular step we take 31 radial steps with $R$ ranging from 2 to $50 \mathrm{a}_{0}$. For small value of $R$ and for $\theta$ close to $0^{\circ}$ or $180^{\circ}$, the $\mathrm{He}$ atom is very close to one end of the $\mathrm{C}_{3} \mathrm{~N}$ molecule and thus the interaction energy becomes extremely large. Fitting correctly this dramatic variation of the interaction energy would require a very large angular basis set, not to mention, that in some cases, the $\operatorname{CCSD}(\mathrm{T})$ calculation failed. We therefore apply an energy cut-off of $28000 \mathrm{~cm}^{-1}$ to overcome these difficulties. Such a large value of the cut-off energy is not expected to affect the collisional dynamics at low temperatures.

\subsubsection{Analytical representation}

The $a b$ initio points were fitted to the following functional form expanded on products of radial and angular functions:

$V(R, \theta)=\sum_{l=0}^{20} v_{l}(R) P_{l}(\cos \theta)$

where the $P_{l}$ are Legendre polynomials. The radial part is made of two terms associated respectively with the short-range and longrange contributions:

$v_{l}(R)=w(R) f_{l}^{(\mathrm{SR})}(R)+[1-w(R)] f_{l}^{(\mathrm{LR})}(R)$

where the switching function $w(R)$ is an hyperbolic tangent. The short-range contribution $f_{l}^{(\mathrm{SR})}(R)$ was interpolated using a cubic spline method, whereas the long-range part $f_{l}^{(\mathrm{LR})}(R)$ was fitted to reciprocal power functions:

$f_{l}^{(\mathrm{LR})}(R)=\sum_{k=6}^{8} \frac{t_{k}(\beta R)}{R^{k}} C_{l k}$

where $t_{k}$ is the Tang-Toennies damping function:

$t_{k}(x)=1-e^{-x} \sum_{i=0}^{k} \frac{x^{i}}{i !}$

The accuracy of the fitting procedure was checked by calculating the root mean square (RMS) of the fitting error for different regions of the PES. In the attractive region $(E<0)$ the RMS is 0.008 $\mathrm{cm}^{-1}$ while in the long-range region $\left(R \geq 14 a_{0}\right)$ the RMS is $0.0009 \mathrm{~cm}^{-1}$. The largest differences are observed in the repulsive part of the PES $(E>0)$ where for instance the RMS is 0.2353 $\mathrm{cm}^{-1}$ for energies below $5000 \mathrm{~cm}^{-1}$.

\subsection{Energy levels of $\mathrm{C}_{3} \mathrm{~N}$}

The electronic and nuclear spin of $\mathrm{C}_{3} \mathrm{~N}$ are both non-zero. Thus, its rotational energy levels exhibit both fine and hyperfine structure as shown in Figure 1. The energy levels represented in this figure show that the fine and hyperfine spacings are much smaller than the rotational ones, therefore ensuring the accuracy of the recoupling scheme.

The effective Hamiltonian used by Gottlieb et al. (1983) to describe the electronic ground state of $\mathrm{C}_{3} \mathrm{~N}\left({ }^{2} \Sigma\right)$ is:

$$
\begin{aligned}
H_{\mathrm{eff}} & =B \boldsymbol{N}^{2}-D \boldsymbol{N}^{4}+\gamma(\boldsymbol{N} \cdot \boldsymbol{S})+\gamma_{D}(\boldsymbol{N} \cdot \boldsymbol{S}) \boldsymbol{N}^{2}+b(\boldsymbol{I} \cdot \boldsymbol{S})+c\left(I_{z} \cdot S_{z}\right) \\
& +\frac{1}{2} \frac{e q Q}{I(2 I-1) J(2 J-1)}\left[3(\boldsymbol{I} \cdot \boldsymbol{J})^{2}+\frac{3}{2}(\boldsymbol{I} \cdot \boldsymbol{J})-\boldsymbol{I}^{2} \boldsymbol{J}^{2}\right]
\end{aligned}
$$

where $\boldsymbol{N}, \boldsymbol{S}, \boldsymbol{I}$ and $\boldsymbol{J}$ are respectively the rotational, electronic, nuclear spin and total angular momentum excluding nuclear spin. The molecular constants $B, D, \gamma, \gamma_{D}, b, c, e q Q$ are set to the values recommended by Gottlieb et al. (1983). Since the spin-rotation interaction is larger than the nuclear hyperfine interactions the coupling scheme $\left(b_{\beta J}\right)$ :

$N+S=J, \quad J+I=F$

is expected to give an accurate description of the molecular wavefunctions. The matrix elements of the $H_{\mathrm{eff}}$ for a coupling scheme $\left(b_{\beta J}\right)$ can be found in Townes \& Schawlow (2013).

\subsection{Scattering formalism}

\subsubsection{Fine resolved cross-section and rate coefficient}

The quantum scattering formalism for the collision of a ${ }^{2} \Sigma$ molecule with a closed shell atom has been described in detail long ago by Alexander (1982). Since we are interested in collision energies quite large compared to the fine structure splitting, this exact approach is however not needed and we use instead the recoupling method to obtain the fine structure resolved state-to-sate cross sections, as well as the IOSA and MRL approximate methods.

2.3.1.1 Recoupling. Within this approach introduced by Corey \& McCourt (1983), the conventional coupling scheme

$\boldsymbol{N}+\boldsymbol{S}=\boldsymbol{J}, \quad \boldsymbol{J}+\boldsymbol{L}=\boldsymbol{R}$

is replaced by

$N+L=Q, \quad Q+S=R$

where $\boldsymbol{L}$ and $\boldsymbol{R}$ are respectively the relative and total angular momenta of the system. In equation (8) the intermediate angular momentum $\boldsymbol{Q}$ is identical to the total angular momentum for an atom colliding with a ${ }^{1} \Sigma$ molecule. Using the fact that the interaction potential is independent of the spin, Corey \& McCourt (1983) obtained the following relation between the $\boldsymbol{T}$ matrices associated with the coupling schemes (7) and (8).

$T_{N J L, N^{\prime} J^{\prime} L^{\prime}}^{R}=(-1)^{N-N^{\prime}+L-L^{\prime}}\left\{[J]\left[J^{\prime}\right]\right\}^{1 / 2}$

$\times \sum_{Q}[Q]\left\{\begin{array}{lll}S & N & J \\ L & R & Q\end{array}\right\}\left\{\begin{array}{ccc}S & N^{\prime} & J^{\prime} \\ L^{\prime} & R & Q\end{array}\right\} T_{N L, N^{\prime} L^{\prime}}^{Q}$

where $[X] \equiv(2 X+1)$. The spin selected state-to-state $\boldsymbol{T}$ matrices are deduced using simple algebra from those calculated when neglecting the spin. Therefore we perform the calculations as if $\mathrm{C}_{3} \mathrm{~N}$ were a ${ }^{1} \Sigma$ molecule and obtain the state-to-state integral cross section from

$\sigma_{N J \rightarrow N^{\prime} J^{\prime}}=\frac{\pi}{k_{N J}^{2}[J]} \sum_{R}[R] \sum_{L L^{\prime}}\left|T_{N J L, N^{\prime} J^{\prime} L^{\prime}}^{R}\right|^{2}$

where $k_{N J}$ is the initial relative wave number in the $N J$ channel. 


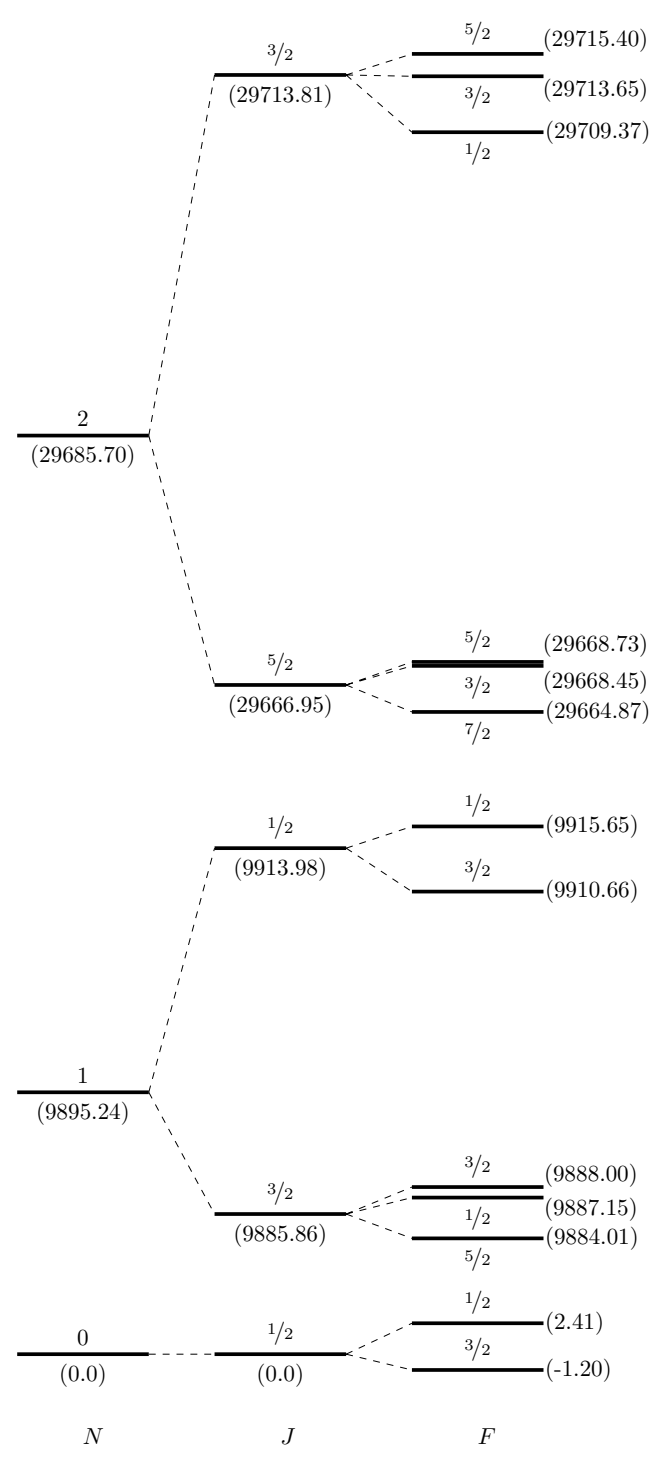

Figure 1. Energy levels diagram. Numbers in parenthesis give the energy in $\mathrm{MHz}$

2.3.1.2 IOSA. For collision energies larger than the rotational spacing, the rotational motion can be neglected, therefore allowing to apply the IOSA. The following scaling relation (Corey \& McCourt 1983) can be used:

$$
\begin{aligned}
\sigma_{N J \rightarrow N^{\prime} J^{\prime}}= & \frac{k_{0}^{2}}{k_{N J}^{2}}[N]\left[N^{\prime}\right]\left[J^{\prime}\right] \sum_{X}\left(\begin{array}{ccc}
N^{\prime} & N & X \\
0 & 0 & 0
\end{array}\right)^{2} \\
& \times\left\{\begin{array}{rrr}
N & N^{\prime} & X \\
J^{\prime} & J & S
\end{array}\right\}^{2} \sigma_{N=0 \rightarrow X}
\end{aligned}
$$

where $k_{0}$ is the spin-free wave number for the state $N=0$ while $\sigma_{N=0 \rightarrow X}$ are the spin-free state-to-state inelastic cross section from $N=0$ towards the final rotational state $N=X$.

2.3.1.3 MRL. This simple approach was first introduced by Alexander \& Dagdigian (1985) for transitions between hyperfine levels and is here equivalently applied to transitions between fine levels. Within this model, the relative angular momentum of the collision partners is neglected and the fine resolved cross sections are assumed to be purely statistical, proportional to the degeneracy of the final state and independent of the initial hyperfine level:

$\sigma_{N J \rightarrow N^{\prime} J^{\prime}}=\frac{\left[J^{\prime}\right]}{[S]\left[N^{\prime}\right]} \sigma_{N \rightarrow N^{\prime}}$

where $\sigma_{N \rightarrow N^{\prime}}$ are the spin-free cross sections. This approach is expected to work for very weak interactions and to fail for quasielastic transitions $N=N^{\prime}, J \neq J^{\prime}$

\subsubsection{Hyperfine resolved cross-section and rate coefficient}

Since the hyperfine splitting is even smaller than the fine one and because the interaction potential can be safely assumed to be also independent of the nuclear spin, the same approximations can be used.

2.3.2.1 Recoupling. As seen above, the transformation between the coupling schemes:

$\boldsymbol{J}+\boldsymbol{I}=\boldsymbol{F}, \quad \boldsymbol{F}+\boldsymbol{L}=\boldsymbol{K}$

and

$\boldsymbol{J}+\boldsymbol{L}=\boldsymbol{R}, \quad \boldsymbol{R}+\boldsymbol{I}=\boldsymbol{K}$

leads to the following relation

$T_{N J F L, N^{\prime} J^{\prime} F^{\prime} L^{\prime}}^{K}=(-1)^{J-J^{\prime}+L-L^{\prime}}\left\{[F]\left[F^{\prime}\right]\right\}^{1 / 2}$

$\times \sum_{R}[R]\left\{\begin{array}{ccc}I & J & F \\ L & K & R\end{array}\right\}\left\{\begin{array}{ccc}I & J^{\prime} & F^{\prime} \\ L^{\prime} & K & R\end{array}\right\} T_{N J L, N^{\prime} J^{\prime} L^{\prime}}^{R}$

and using (9), the hyperfine state-to-state $\boldsymbol{T}$ matrix is deduced from the one calculated when neglecting the nuclear and electronic spin by

$$
\begin{aligned}
& T_{N J F L, N^{\prime} J^{\prime} F^{\prime} L^{\prime}}^{K}=(-1)^{J-J^{\prime}+N-N^{\prime}}\left\{[J]\left[J^{\prime}\right][F]\left[F^{\prime}\right]\right\}^{1 / 2} \\
& \times \sum_{Q}[Q] T_{N L, N^{\prime} L^{\prime}}^{Q} \sum_{R}[R]\left\{\begin{array}{ccc}
I & J & F \\
L & K & R
\end{array}\right\}\left\{\begin{array}{ccc}
I & J^{\prime} & F^{\prime} \\
L^{\prime} & K & R
\end{array}\right\} \\
& \times\left\{\begin{array}{ccc}
S & N & J \\
L & R & Q
\end{array}\right\}\left\{\begin{array}{ccc}
S & N^{\prime} & J^{\prime} \\
L^{\prime} & R & Q
\end{array}\right\}
\end{aligned}
$$

where the good quantum numbers $I$ and $S$ are implicit in the notation of the $\boldsymbol{T}$ matrix elements. Since the summation over $R$ can be represented as a $12 j$-symbol of the second kind, see equation (25) in Appendix, this relation can be rewritten in a more concise form as

$$
\begin{aligned}
& T_{N J F L, N^{\prime} J^{\prime} F^{\prime} L^{\prime}}^{K}=(-1)^{J-J^{\prime}-F+F^{\prime}}\left\{[J]\left[J^{\prime}\right][F]\left[F^{\prime}\right]\right\}^{1 / 2} \\
& \times \sum_{Q}[Q]\left\{\begin{array}{cccc}
- & L^{\prime} & Q & N^{\prime} \\
J & - & N & S \\
I & F^{\prime} & - & J^{\prime} \\
F & K & L & -
\end{array}\right\} T_{N L, N^{\prime} L^{\prime}}^{Q}
\end{aligned}
$$

The hyperfine resolved integral cross-section are then calculated as

$\sigma_{N J F \rightarrow N^{\prime} J^{\prime} F^{\prime}}=\frac{\pi}{k_{N J F}^{2}[F]} \sum_{K}[K] \sum_{L L^{\prime}}\left|T_{N J F L, N^{\prime} J^{\prime} F^{\prime} L^{\prime}}^{K}\right|^{2}$

2.3.2.2 IOSA. Using equations (13) and (17) from Alexander \& Dagdigian (1985), the hyperfine resolved cross section are obtained 
as

$$
\begin{aligned}
\sigma_{N J F \rightarrow N^{\prime} J^{\prime} F^{\prime}=} & \frac{k_{0}^{2}}{k_{N J F}^{2}}[N]\left[N^{\prime}\right][J]\left[J^{\prime}\right]\left[F^{\prime}\right] \sum_{X}\left(\begin{array}{ccc}
N & N^{\prime} & X \\
0 & 0 & 0
\end{array}\right)^{2} \\
& \times\left\{\begin{array}{ccc}
J & J^{\prime} & X \\
F^{\prime} & F & I
\end{array}\right\}^{2}\left\{\begin{array}{rrr}
N & N^{\prime} & X \\
J^{\prime} & J & S
\end{array}\right\}^{2} \sigma_{N=0 \rightarrow X}
\end{aligned}
$$

where $I$ and $S$ are again implicit in the notation of the cross-section.

2.3.2.3 MRL. Following the guidelines described in the work of Alexander \& Dagdigian (1985), the hyperfine resolved cross section can be obtained directly from the spin-free cross section as

$\sigma_{N J F \rightarrow N^{\prime} J^{\prime} F^{\prime}}=\frac{[J]\left[J^{\prime}\right]\left[F^{\prime}\right]}{\left[N^{\prime}\right]}\left\{\begin{array}{cccc}- & N & J & S \\ F^{\prime} & - & I & J^{\prime} \\ J^{\prime} & S & - & N^{\prime} \\ I & J & F & -\end{array}\right\} \sigma_{N, N^{\prime}}$

From all the previously defined cross sections, the thermally averaged rate coefficients are obtained by

$k_{i \rightarrow f}(T)=\sqrt{\frac{8}{\pi \mu}}\left(k_{\mathrm{B}} T\right)^{-\frac{3}{2}} \int_{0}^{\infty} \sigma_{i \rightarrow j}(E) \mathrm{e}^{-\frac{E}{k_{\mathrm{B}} T}} E d E$

\subsubsection{Parameters of the Close Coupling calculations}

In order to obtain the $T_{N L, N^{\prime} L^{\prime}}^{Q}$ matrix elements required in equation (9), we solve the Close Coupling equations using the logderivative propagator (Manolopoulos 1986) implemented in the NEWMat code. As the value of the rotational constant $B$ of $\mathrm{C}_{3} \mathrm{~N}$ is small, a total of 40 rotational levels was needed in the rotational basis set used for the calculation of the dynamics. The calculations are performed for a grid of collision energies ranging from 0.1 up to $2000 \mathrm{~cm}^{-1}$. For each collision energy, the convergence of the spin-free de-excitation cross sections was checked as a function of the maximal value of the total angular momentum quantum number $Q$ which was 150 for the highest collision energy.

\section{Results}

\subsection{Interaction potential}

The PES is characterized by a large angular anisotropy and a shallow potential well. The global minimum, with the dissociation energy $D_{e}=43.43 \mathrm{~cm}^{-1}$, is associated with a near T-shaped geometry $\left(R=6.26 a_{0}, \theta=86.81^{\circ}\right)$. Two linear saddle points are found: one with the $\mathrm{He}$ atom facing the $\mathrm{N}$-end $\left(R=9.86 a_{0}, \theta=0^{\circ}\right)$ while in the other one the He atom faces the C-end $\left(R=10.12 a_{0}\right.$, $\left.\theta=180^{\circ}\right)$. They both connects two equivalents configurations of the global minimum and their energy relative to the global minimum are respectively $22.30 \mathrm{~cm}^{-1}$ and $22.80 \mathrm{~cm}^{-1}$. These features are depicted in Figure 2.

\subsubsection{Long-range interactions}

There is no electrostatic contribution to the long range potential as all the multipole moments of He are zero. The long-range potential is then restricted to the sum of the induction and dispersion contributions

$V=V_{\text {ind }}+V_{\text {disp }}$

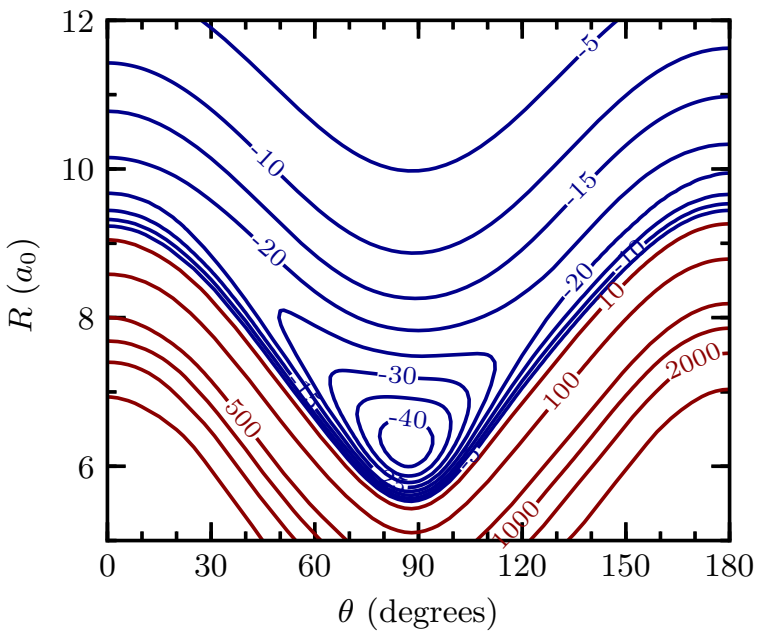

Figure 2. 2D contour plots of the potential energy surface. Contour levels are labeled by the energy in $\mathrm{cm}^{-1}$. The contours are blue for negative interaction energy, and red for positive one

Table 1. Asymptotic form of the leading expansion coefficients as defined in equation (1) for the long-range interaction contributions. $\mu$ and $\Theta$ are the dipole and quadrupole moment respectively. $\alpha=\frac{1}{3}\left(\alpha_{\|}+2 \alpha_{\perp}\right)$ and $\Delta \alpha=\left(\alpha_{\|}-\alpha_{\perp}\right)$ are the isotropic and anisotropic polarizabilities where $\alpha_{\|}$ and $\alpha_{\perp}$ are respectively the parallel and perpendicular dipole polarizabilities. $A_{\|}$and $A_{\perp}$ are dipole-quadrupole polarizabilities and $U$ the ionization energies. Subscripts $a$ and $b$ refer to $\mathrm{He}$ and $\mathrm{C}_{3} \mathrm{~N}$ respectively. The multipole moments and polarizabilities values have been calculated with the origin at

\begin{tabular}{|c|c|c|c|c|c|c|c|}
\hline$v_{l}$ & \multicolumn{4}{|c|}{ Induction } & \multicolumn{3}{|c|}{ Dispersion } \\
\hline \multirow{5}{*}{$\begin{array}{l}v_{0} \\
v_{1} \\
v_{2} \\
v_{3}\end{array}$} & \multirow{4}{*}{\multicolumn{4}{|c|}{$\begin{array}{c}-\frac{\mu_{b}^{2} \alpha_{a}}{R^{6}}+\ldots \\
-\frac{12 \sqrt{3}}{5} \frac{\alpha_{a} \mu_{b} \Theta_{b}}{R^{7}}+\ldots \\
-\frac{2 \sqrt{5}}{5} \frac{\alpha_{a} \mu_{b}^{2}}{R^{6}}+\ldots \\
\frac{24 \sqrt{7}}{35} \frac{\alpha_{a} \mu_{b} \Theta_{b}}{R^{7}}+\ldots\end{array}$}} & \multirow{4}{*}{\multicolumn{3}{|c|}{$\begin{array}{r}-3 \frac{U_{a} U_{b}}{U_{a}+U_{b}} \frac{\alpha_{a} \alpha_{b}}{R^{6}}+\ldots \\
-\frac{6 \sqrt{3}}{5} \frac{U_{a} U_{b}}{U_{a}+U_{b}} \frac{\alpha_{a}\left(A_{\|}+2 A_{\perp}\right)_{b}}{R^{7}} \\
-\frac{\sqrt{5}}{5} \frac{U_{a} U_{b}}{U_{a}+U_{b}} \frac{\alpha_{a} \Delta \alpha_{b}}{R^{6}}+\ldots \\
\frac{4 \sqrt{7}}{37} \frac{U_{a} U_{b}}{U_{a}+U_{b}} \frac{\alpha_{a}\left(4 A_{\|}-3 A_{\perp}\right)_{b}}{R^{7}}\end{array}$}} \\
\hline & & & & & & & \\
\hline & & & & & & & \\
\hline & & & & & & & \\
\hline & $\mu$ & $\Theta$ & $\alpha_{\|}$ & $\alpha_{\perp}$ & $A_{\|}$ & $A_{\perp}$ & $U$ \\
\hline $\mathrm{C}_{3} \mathrm{~N}$ & -1.13 & -1.13 & 60.35 & 27.11 & -25.05 & -32.52 & $0.53^{\dagger}$ \\
\hline $\mathrm{He}$ & 0 & 0 & 1.38 & 1.38 & 0 & & $0.90^{\ddagger}$ \\
\hline
\end{tabular}
the center of mass. All quantities are given in atomic units.

$\dagger$ Dibeler et al. (1961)

‡ Lide (1992)

where each term is in turn expanded as in equation (1). The analytical forms of the leading expansion coefficients for each contributions are well known (Buckingham 1978) and reminded in Table 1.

The multipole moments, polarizabilities and ionization energies used in the present work are also given in Table 1 . They were calculated using the finite field method implemented in MOLPRO at the $\operatorname{CCSD}(\mathrm{T}) /$ aug-cc-pVTZ level.

A good agreement is obtained between the long-range fitted coefficients of the PES and their calculated values using the formulae of Table 1, as seen in Figure 3. The small differences found arise from both the truncation of the analytic expansion and the inaccuracy of the London's approximation of the dispersion contribution. Therefore, we assume that the present PES is suitable for describing cold molecular collisions. The largest contribution appearing in Figure 3 is due to the isotropic induction + dispersion term $v_{0}$ which is proportional to $R^{-6}$, see Table 1 . The ratio of the dispersion to 


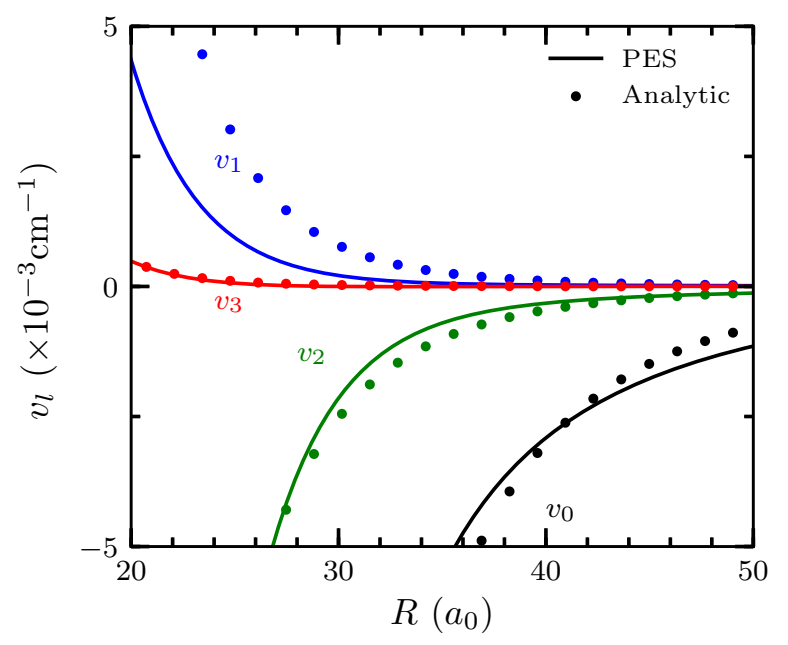

Figure 3. Comparison between the two methods of calculation of the long range expansion coefficients $v_{l}(R)$. The coefficients are obtained from the fit of the $a b$ initio data (solid lines) or by the formulae of Table 1 (solid circles).

induction contributions for the $v_{0}$ term is

$3 \frac{U_{a} U_{b}}{U_{a}+U_{b}} \frac{\alpha_{b}}{\mu_{b}^{2}}=30.102$

Dispersion is then 30 times stronger than induction and the longrange potential is thus dominated by dispersion.

\subsection{Rate coefficients}

Using the three approaches described in section 2.3, we computed fine and hyperfine resolved rate coefficients for $\mathrm{C}_{3} \mathrm{~N}$ colliding with $\mathrm{He}$ in the [10-500] $\mathrm{K}$ temperature range. We considered all the $N \rightarrow N^{\prime}$ transitions of $\mathrm{C}_{3} \mathrm{~N}$ for $0 \leq N \leq 39$ and $0 \leq N^{\prime} \leq 39$. In the absence of any Close Coupling calculations available for this system, we compare our recoupling results with those obtained using both the IOSA and MRL approximations in Figures 4 and 5 respectively for the fine and hyperfine resolved transitions.

In these figures the MRL approximation is seen to fail at reproducing the fine and hyperfine resolved rate coefficients over the whole temperature range. However, for some transitions and specially those with $N=N^{\prime}=0$, the agreement is remarkable. The IOSA, on the other hand, predicts the rate coefficients moderately well at higher temperatures whereas the agreement at low temperatures is not so good. This is not surprising since the IOSA is expected to fails at low collision energies and therefore low temperatures. We then suggest that the IOSA should be preferred to the MRL approximation as it not only gives very reliable results but also requires less input data when compared to MRL.

\subsubsection{Fine structure resolved rate coefficients}

The variation with temperature of the fine structure resolved deexcitation rate coefficients of the $N=5$ multiplet of $\mathrm{C}_{3} \mathrm{~N}$ are presented in Figure 6. The rate coefficients are seen to increase at low temperatures while being almost constant at higher temperatures. They follow the typical $\Delta J=\Delta N$ propensity rule which was investigated by Alexander \& Dagdigian (1985) and Corey \&
Alexander (1988), along with a similar propensity rule for hyperfine resolved rates. Since the atom-molecule interaction potential is purely electrostatic and no external magnetic fields are considered, then the electronic and nuclear spins play a spectator role in the collisions. Alexander \& Dagdigian (1985) have shown that the $\Delta J=\Delta N$ propensity rule can be understood in term of vector coupling argument and is therefore independent of the interaction potential. Consequently, the latter propensity rule has been observed in various systems (Dumouchel et al. 2012; Dagdigian 2020).

Even $\Delta N$ and $\Delta J$ are also favoured when $\Delta J=\Delta N$. When $\Delta N \neq \Delta J$, the odd $\Delta J$ are enhanced because the $\mathrm{C}_{3} \mathrm{~N}$ electronic spin is $S=\frac{1}{2}$ and thus the only possibles values of $\Delta J$ are $\Delta N \pm 1$ which are both odd numbers. As a particular case of the even $\Delta J=\Delta N$ propensity rule, we observe also a $\Delta J=\Delta N=0$ propensity rule which is in agreement with the propension to keep $J$ unchanged as discussed by Alexander \& Dagdigian (1985).

The $\Delta N$ even propensity rule is characteristic of nearly homonuclear symmetric molecules as shown by McCurdy \& Miller (1977). This is indeed what can be seen in Figure 1,where the interaction potential appears to be almost symmetrical with respect to the $\theta=90^{\circ}$ axis. The Legendre polynomial even expansion coefficients of the potential are consequently larger than the odd ones, as shown in Figure 3. We found no evidence of any occurrence of a propensity inversion but found that the $\Delta N$ even propensity rule tend to disappear for large $\Delta N$ values.

\subsubsection{Hyperfine resolved rate coefficients}

The temperature dependence of the hyperfine resolved de-excitation rate coefficients is presented in Figure 8 for the same $N=5$ multiplet of $\mathrm{C}_{3} \mathrm{~N}$. The rate coefficients are again seen to be almost constant at high temperatures. Additionally to the previously discussed $\Delta J=$ $\Delta N$ propensity rule, the hyperfine resolved transitions display a similar $\Delta F=\Delta J$ propensity rule, which results from the fact that the nuclear spin cannot be reoriented during the collision. Since this propensity rule is independent of the interaction potential, it has been previously observed in many systems (Ben Abdallah et al. 2012; Dumouchel et al. 2012; Kalugina et al. 2012; Marinakis et al. 2019; Dagdigian 2020; Kłos et al. 2020). We also observe in Figure 9 the strong even $\Delta N$ propensity rule discussed in the previous section, as well as a $\Delta F=\Delta J=\Delta N=0$ propensity rule. More generally, we conclude from a careful inspection of this figure that the rate coefficients for fine and hyperfine transitions follow the $(\Delta F=\Delta J=\Delta N)>(\Delta J=\Delta N)>($ even $\Delta N)$ propensity rule. A set of hyperfine resolved rate coefficients is provided in the supplementary material.

\subsubsection{Rate coefficients for collisions with $\mathrm{H}_{2}$}

$\mathrm{H}_{2}$ is much more abundant than $\mathrm{He}$ in interstellar clouds and it is generally the dominant collision partner. The rate coefficients for the (de)excitations of a any molecule $\mathrm{X}$ by collision with $\mathrm{He}$ is expected to provide an estimate for those with para- $\mathrm{H}_{2}$ by using the mass-scaling law (Schöier et al. 2005)

$k_{\mathrm{H}_{2}}(T)=k_{\mathrm{He}}(T) \sqrt{\frac{\mu_{\mathrm{He}-\mathrm{X}}}{\mu_{\mathrm{H}_{2}-\mathrm{X}}}}$

where $\mu_{\mathrm{A}-\mathrm{X}}$ stands for the reduced mass of the $\mathrm{A}+\mathrm{X}$ collisional system. This approximation is valid only for $\mathrm{H}_{2}$ in its rotational ground state $j=0$ and is based on the assumption that the cross sections with $\mathrm{He}$ and $\mathrm{H}_{2}$ are close. When the molecule $\mathrm{X}$ is much heavier than $\mathrm{He}$ or $\mathrm{H}_{2}$, then the scaling factor is close to 1.4. However, 

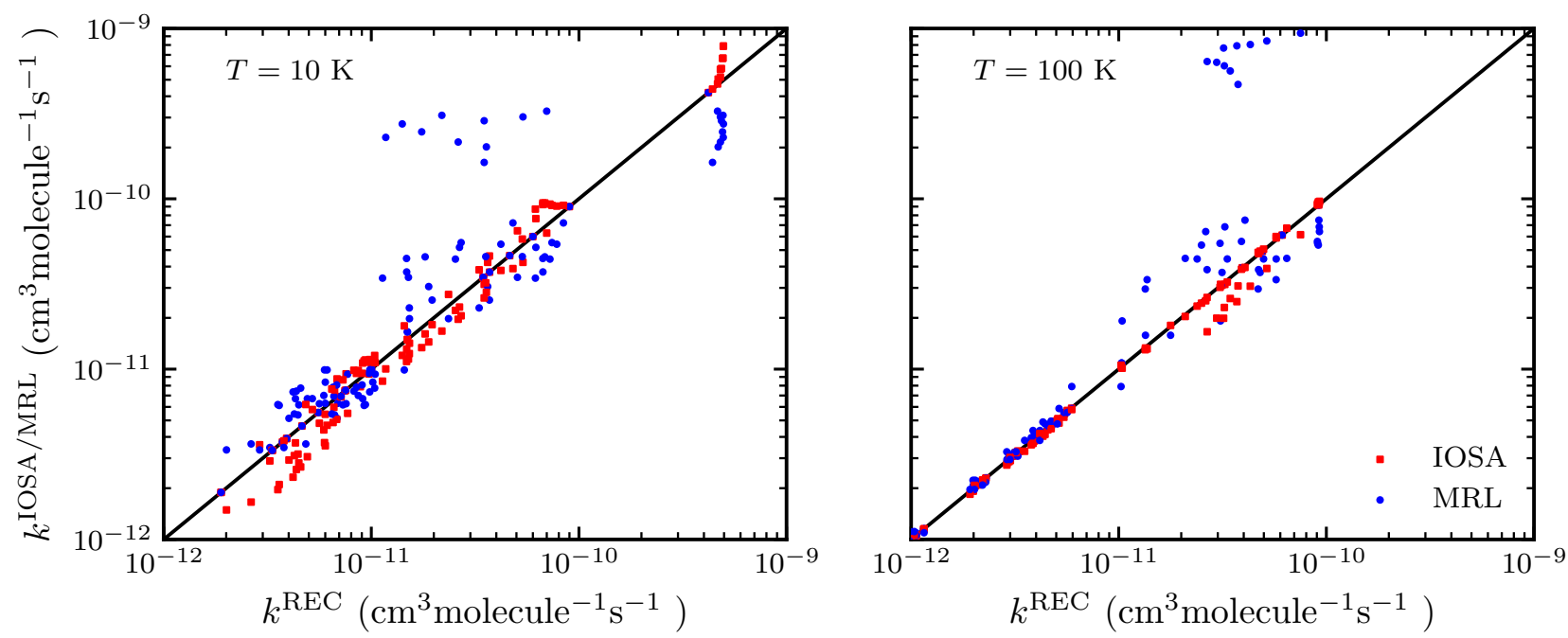

Figure 4. Comparison between the fine resolved rate coefficients calculated with the recoupling, IOSA, and MRL methods. We have included all the transitions between the $\mathrm{C}_{3} \mathrm{~N}$ energy levels with $N$ and $N^{\prime} \in[0,5]$.
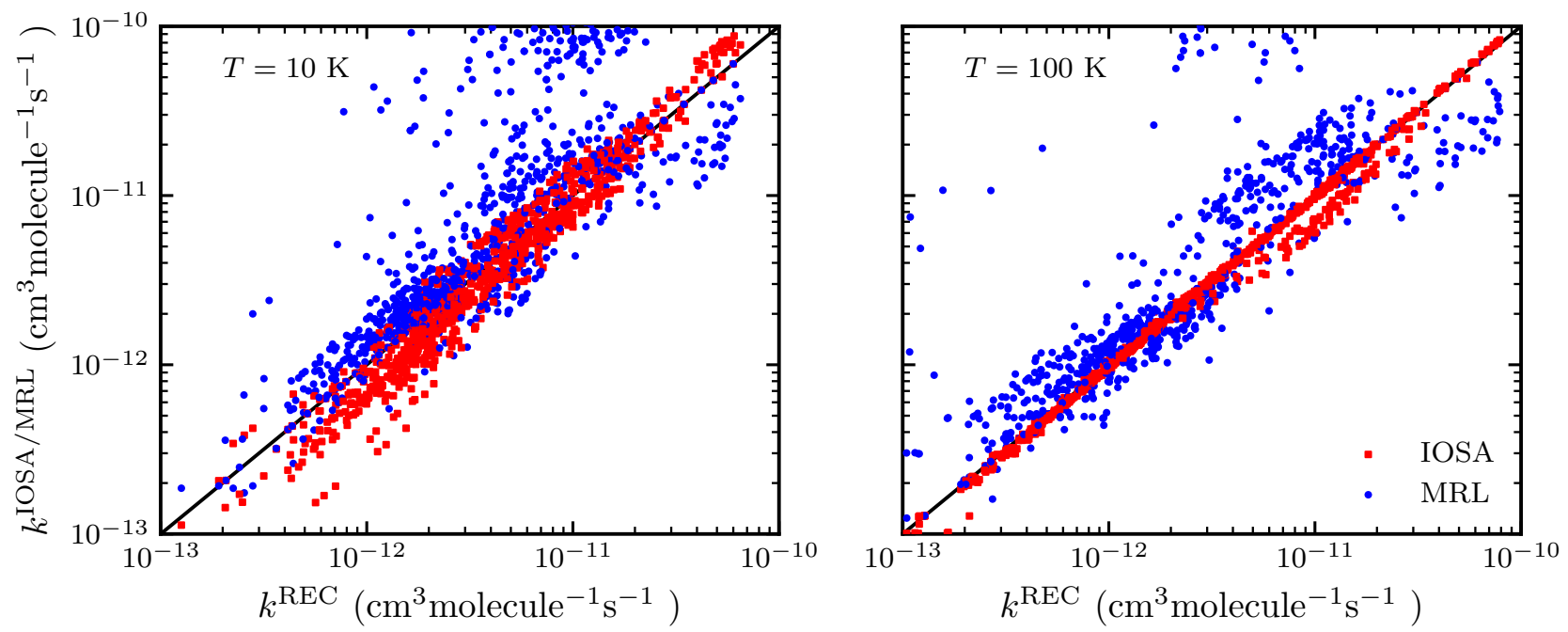

Figure 5. Comparison between the hyperfine resolved rate coefficients calculated with the recoupling, IOSA, and MRL methods. We have included all the transitions between the $\mathrm{C}_{3} \mathrm{~N}$ energy levels with $N$ and $N^{\prime} \in[0,5]$.

there is some evidence that the cross sections could be significantly different. $\mathrm{H}_{2}$ has a quadrupole moment while $\mathrm{He}$ has no multipole moment. Therefore, the long range interaction between a dipolar molecule and $\mathrm{H}_{2}$ scales as $R^{-4}$, while with $\mathrm{He}$, the dominant long range interactions are the dipole-induced dipole and the dispersion which scale as $R^{-5}$ and $R^{-6}$, respectively. Furthermore, the polarizability of $\mathrm{H}_{2}$ is four times larger than the one of He. Consequently, the cross sections at low energy with the collider $\mathrm{H}_{2}$ are expected to be larger than the one with He. Some other properties of the interaction energy can also produce large differences between cross-sections, such as the depth of the potential well which is generally much larger with $\mathrm{H}_{2}$ than with $\mathrm{He}$, and the structure of the equilibrium geometry which can be linear in one case and T-shaped in the other case.

The validity of mass-scaling has been examined for various molecular systems, namely $\mathrm{X}=\mathrm{SiS}$ (Lique et al. 2008), $\mathrm{HCN}$ (Ben Abdallah et al. 2012), HF (Guillon \& Stoecklin 2012), CO (Walker et al. 2014), CS (Denis-Alpizar et al. 2018), $\mathrm{C}_{3} \mathrm{~N}^{-}$(LaraMoreno et al. 2019b). The ratio of the two sets of rate coefficients, with $\mathrm{He}$ or with $\mathrm{H}_{2}(j=0)$, varies both with the temperature and the transition considered. As long as we consider the dominant rate coefficients, the rates for $\mathrm{H}_{2}(j=0)$ are generally larger by a factor between 1 and 3 than the corresponding rates with $\mathrm{He}$, and in some cases, by about one order of magnitude. For the small rate coefficients, larger differences can be observed. The propensity rules observed in collisions with $\mathrm{H}_{2}$ are generally not reproduced correctly by the mass-scaling procedure and moreover, spurious propensity rules can be generated (Lara-Moreno et al. 2019b). However, in the case of the hyperfine resolved rates for the transitions of $\mathrm{C}_{3} \mathrm{~N}$, since the propensity rules are mostly independent of the interaction 


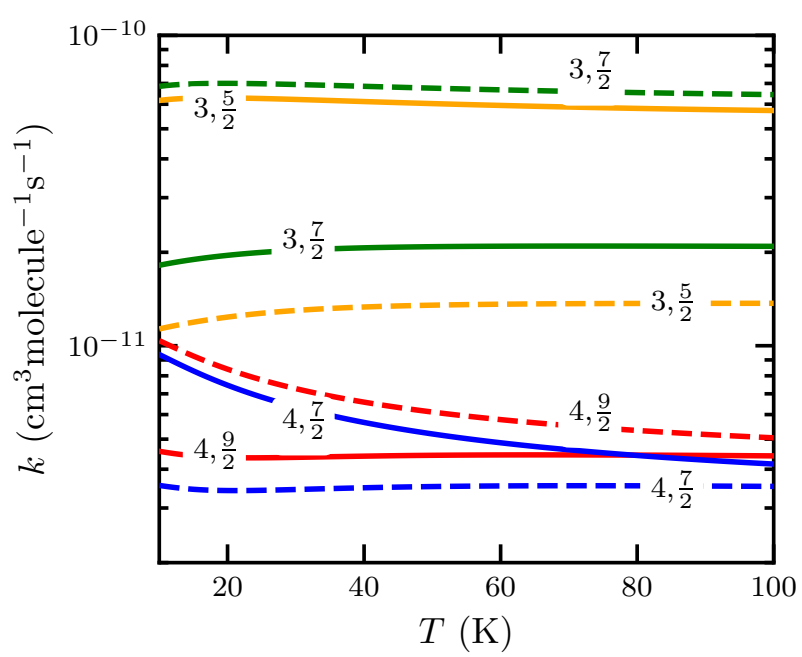

Figure 6. Fine resolved de-excitation rate coefficients of $\mathrm{C}_{3} \mathrm{~N}(J, N=5)$. Solid lines correspond to transitions with initial state $J=\frac{9}{2}$ while dashed lines are those with $J=\frac{11}{2}$. The curves are labeled with the final $N^{\prime}, J^{\prime}$ states.

potential, they should be similar for the two sets of rate coefficients. In summary, mass-scaling provides only a poor estimate of the rate coefficients for collisions with $\mathrm{H}_{2}(j=0)$, but at least within an order of magnitude of the rates obtained by accurate close coupling calculation. Nevertheless, when it is the single source of rate coefficients for transitions induced by collisions with $\mathrm{H}_{2}(j=0)$, mass-scaling might be useful. We provide a set of rate coefficients calculated with the equation 24 in the supplementary material.

\section{Conclusions}

We presented the first Close Coupling study of the rotational excitation of $\mathrm{C}_{3} \mathrm{~N}$ by collision with helium within the rigid rotor approximation. To this aim a 2D ab initio PES was computed. The $\mathrm{He}-\mathrm{C}_{3} \mathrm{~N}$ complex is found to be nearly $\mathrm{T}$ shaped with a very shallow well of $43.43 \mathrm{~cm}^{-1}$ while dispersion is shown to give the main contribution to the long-range potential. The rate coefficients given by the IOSA and MRL approximations are compared with those of the recoupling method. It is found that the IOSA should be preferred to the MRL approximation for this system as the reliability of MRL is unpredictable. The fine structure resolved rate coefficients follow the $\Delta J=\Delta N$ propensity rule predicted by Alexander \& Dagdigian (1985) which results from the fact that the electronic spin cannot be reoriented in a molecular collision. Even $\Delta N$ and $\Delta J$ are also favoured when $\Delta J=\Delta N$ while odd $\Delta J$ are favoured when $\Delta N \neq \Delta J$. The hyperfine resolved transitions display a similar $\Delta F=\Delta J$ propensity rule, which in this case results from the fact that the nuclear spin cannot be reoriented during the collision. We more generally conclude that the rate coefficients for fine and hyperfine resolved rotational transitions follow the $(\Delta F=\Delta J=\Delta N)$ $>(\Delta J=\Delta N)>($ even $\Delta N)$ rule.

\section{Acknowledgements}

This research has been supported by the Agence Nationale de la Recherche (Project ANR-AnionCosChem). Computer time for this
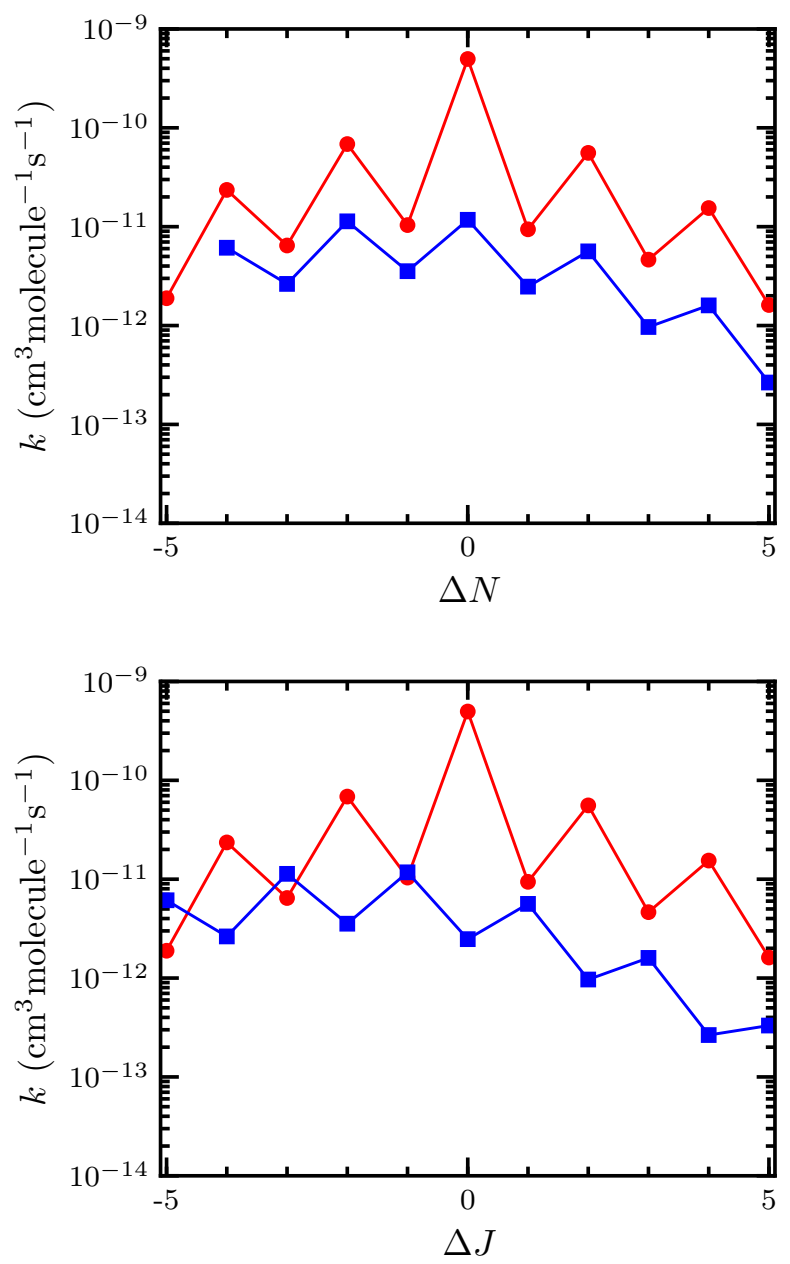

Figure 7. Fine resolved rate coefficients for $\mathrm{C}_{3} \mathrm{~N}\left(N=5, J=\frac{11}{2}\right)$ at $10 \mathrm{~K}$ as a function of the transferred angular momenta $\Delta N$ (top panel) and $\Delta J$ (bottom panel). Red circles correspond to transitions with $\Delta J=\Delta N$ whereas blue squares are transitions with $\Delta J \neq \Delta N$.

study was provided by the computing facilities of the MCIA ( Mésocentre de Calcul Intensif Aquitain).

\section{Data Availability}

The data underlying this article are available in the article and in its online supplementary material.

\section{Appendix. $12 j$-symbol of the second kind}

The $12 j$-symbol of the second kind can be represented as the following sum

$$
\begin{aligned}
& \left\{\begin{array}{cccc}
- & a_{2} & a_{3} & a_{4} \\
b_{1} & - & b_{3} & b_{4} \\
c_{1} & c_{2} & - & c_{4} \\
d_{1} & d_{2} & d_{3} & -
\end{array}\right\}=(-1)^{b_{3}-a_{4}-d_{1}+c_{2}} \sum_{x}[x]\left\{\begin{array}{ccc}
a_{3} & b_{4} & x \\
b_{1} & d_{3} & b_{3}
\end{array}\right\} \\
& \times\left\{\begin{array}{ccc}
a_{3} & b_{4} & x \\
c_{4} & a_{2} & a_{4}
\end{array}\right\}\left\{\begin{array}{lll}
b_{1} & d_{3} & x \\
d_{2} & c_{1} & d_{1}
\end{array}\right\}\left\{\begin{array}{ccc}
c_{4} & a_{2} & x \\
d_{2} & c_{1} & c_{2}
\end{array}\right\}
\end{aligned}
$$




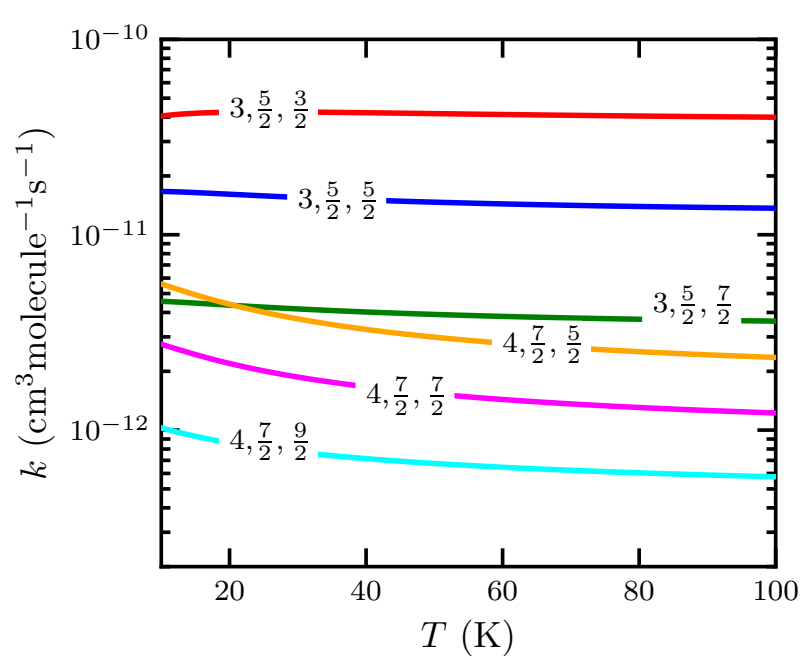

Figure 8. Hyperfine resolved de-excitation rate coefficients of $\mathrm{C}_{3} \mathrm{~N}(N=$ $5, J=\frac{9}{2}, F=\frac{7}{2}$ ). The curves are labeled with the final $N^{\prime}, J^{\prime}, F^{\prime}$ states.

A $12 j$-symbol of the second kind vanishes unless :

- The triangular conditions are fulfilled for the triads $\left(a_{2} a_{3} a_{4}\right)$, $\left(b_{1} b_{3} b_{4}\right), \quad\left(c_{1} c_{2} c_{4}\right), \quad\left(d_{1} d_{2} d_{3}\right), \quad\left(b_{1} c_{1} d_{1}\right), \quad\left(a_{2} c_{2} d_{2}\right), \quad\left(a_{3} b_{3} d_{3}\right)$, $\left(a_{4} b_{4} c_{4}\right)$. The triangular condition for the triad $\left(j_{1} j_{2} j_{3}\right)$ means that $\left|j_{1}-j_{2}\right| \leq j_{3} \leq j_{1}+j_{2}$

- The tetragonal conditions are fulfilled for the tetrads $\left(a_{2} c_{4} d_{3} b_{1}\right),\left(a_{3} b_{4} d_{2} c_{1}\right),\left(a_{4} b_{3} c_{2} d_{1}\right)$. The tetragonal condition for the tetrad $\left(j_{1} j_{2} j_{3} j_{4}\right)$ means that $j_{1}+j_{2}+j_{3}+j_{4}$ is an integer and $j_{1} \leq j_{2}+j_{3}+j_{4}, j_{2} \leq j_{1}+j_{3}+j_{4}, j_{3} \leq j_{1}+j_{2}+j_{4}, j_{4} \leq j_{1}+j_{2}+j_{3}$.

For a more detailed review of the properties of the $12 j$-symbol of second kind, see Varshalovich et al. (1988), Sec. 10.13.3

\section{REFERENCES}

Alexander M. H., 1982, J. Chem. Phys., 76, 3637

Alexander M. H., Dagdigian P. J., 1985, J. Chem. Phys., 83, 2191

Ben Abdallah D., Najar F., Jaidane N., Dumouchel F., Lique F., 2012, MNRAS, 419, 2441

Botschwina P., Horn M., Flügge J., Seeger S., 1993, J. Chem. Soc., Faraday Trans., 89, 2219

Buckingham A. D., 1978, in Pullman B., ed., Intermolecular Interactions: From Diatomics to Biopolymers (Perspectives in Quantum Chemistry \& Biochemistry). John Wiley \& Sons, Inc., New York, pp 1-67

Corey G. C., Alexander M. H., 1988, J. Chem. Phys., 88, 6931

Corey G., McCourt F. R., 1983, J. Phys. Chem., 87, 2723

Cybulski S. M., Toczylowski R. R., 1999, J. Chem. Phys., 111, 10520

Dagdigian P. J., 2020, MNRAS, 498, 5361

Denis-Alpizar O., Stoecklin T., Guilloteau S., Dutrey A., 2018, MNRAS, 478,1811

Dibeler V. H., Reese R. M., Franklin J., 1961, J. Amer. Chem. Soc., 83, 1813 Dumouchel F., Kłos J., Toboła R., Bacmann A., Maret S., Hily-Blant P., Faure A., Lique F., 2012, J. Chem. Phys., 137, 114306

Friberg P., Hjalmarson A., Guelin M., Irvine W. M., 1980, ApJ, 241, L99

Gottlieb C., Gottlieb E., Thaddeus P., Kawamura H., 1983, ApJ, 275, 916

Guillon G., Stoecklin T., 2012, MNRAS, 420, 579

Hoshina K., Endo Y., 2007, J. Chem. Phys., 127, 184304

Kalugina Y., Lique F., Kłos J., 2012, MNRAS, 422, 812

Kłos J., Dagdigian P. J., Alexander M. H., Faure A., Lique F., 2020, MNRAS, 493, 3491

Lara-Moreno M., Stoecklin T., Halvick P., 2017, MNRAS, 467, 4174
Lara-Moreno M., Stoecklin T., Halvick P., 2019a, ACS Earth and Space Chemistry, 3, 1556

Lara-Moreno M., Stoecklin T., Halvick P., 2019b, MNRAS, 486, 414

Lide D. R., 1992, Ionization potentials of atoms and atomic ions. CRC Press Boca Raton, FL

Lique F., Tobola R., Klos J., Feautrier N., Spielfiedel A., Vincent L. F. M., Chalasínski G., Alexander M. H., 2008, A\&A, 478, 567

Manolopoulos D. E., 1986, J. Chem. Phys., 85, 6425

Marinakis S., Kalugina Y., Kłos J., Lique F., 2019, A\&A, 629, A130

McCarthy M., Gottlieb C., Thaddeus P., Horn M., Botschwina P., 1995, J. Chem. Phys., 103, 7820

McCurdy C. W., Miller W. H., 1977, J. Chem. Phys., 67, 463

Schöier F. L., van der Tak F. F. S., van Dishoeck E. F., Black J. H., 2005 , A\&A, 432, 369

Tchakoua T., Motapon O., Nsangou M., 2018, J. Phys. B: At. Mol. Opt. Phys., 51, 045202

Thaddeus P., Gottlieb C. A., Gupta H., Brünken S., McCarthy M. C., Agúndez M., Guélin M., Cernicharo J., 2008, ApJ, 677, 1132

Townes C. H., Schawlow A. L., 2013, Microwave spectroscopy. Dover Publication, Inc., Mineola, New York

Varshalovich D. A., Moskalev A. N., Khersonskii V. K., 1988, Quantum Theory of Angular Momentum. World Scientific, Singapore

Walker K. M., Yang B. H., Stancil P. C., Balakrishnan N., Forrey R. C., 2014, ApJ, 790, 96

Woon D. E., Herbst E., 2009, ApJS, 185, 273

This paper has been typeset from a $\mathrm{T}_{\mathrm{E}} \mathrm{X} / \mathrm{L} \mathrm{AT} \mathrm{E} \mathrm{X}$ file prepared by the author. 

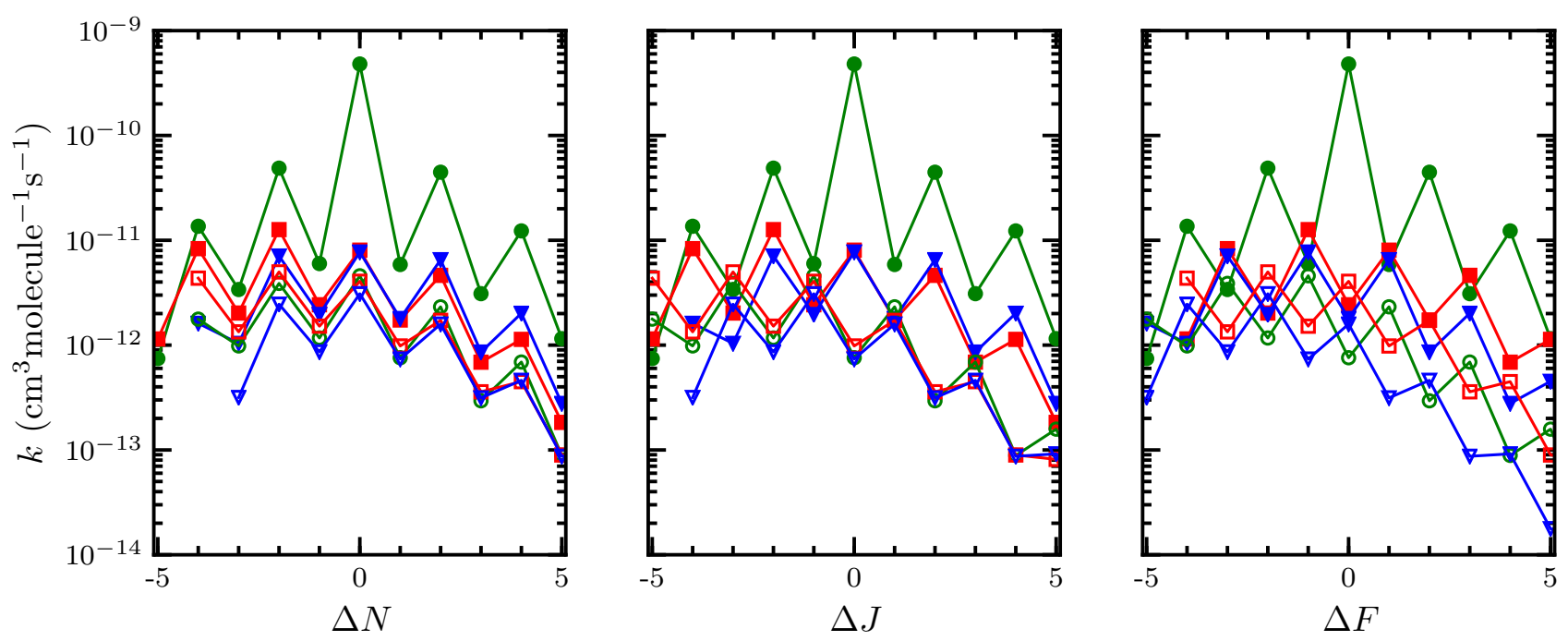

Figure 9. Fine and hyperfine resolved rate coefficients $\mathrm{C}_{3} \mathrm{~N}\left(N=5, J=\frac{9}{2}, F=\frac{7}{2}\right)$ at $10 \mathrm{~K}$ as a function of the transferred angular momenta $\Delta N$ (left panel), $\Delta J$ (middle panel) and $\Delta F$ (right panel). $\Delta F=\Delta J$ transitions are represented by circles, $\Delta F=\Delta J+1$ by squares and $\Delta F=\Delta J-1$ by triangles. Solid symbols correspond to transitions with $\Delta J=\Delta N$ whereas open ones are transitions with $\Delta J \neq \Delta N$. 